\title{
Bi-frequency pendulum 2: modeling apodization, Fourier and adiabatic following in optics
}

\section{B. Zeldovich, M. J. Soileau}

B. Ya. Zeldovich, M. J. Soileau, "Bi-frequency pendulum 2: modeling apodization, Fourier and adiabatic following in optics," Proc. SPIE 9663, Eighth International Topical Meeting on Education and Training in Optics and Photonics, 966321 (6 October 2003); doi: 10.1117/12.2208487

SPIE Event: Eighth International Topical Meeting on Education and Training in Optics and Photonics, 2003, Tucson, Arizona, United States 


\title{
Bi-frequency pendulum 2: modeling apodization, Fourier and adiabatic following in optics
}

\author{
B. Ya. Zeldovich, M. J. Soileau \\ School of Optics / CREOL, University of Central Florida; 4000 Central Florida Blvd, Orlando, FL 32816-2700 \\ Voice (407) 823-6831, FAX (407) 823-6880, boris@creol.ucf.edu
}

\begin{abstract}
Teaching optical phenomena with the use of analogies to the motion of bi-frequency pendulum on a rotary platform is described. Adiabatic following in liquid crystals and in quantum mechanics is visualized. Particular examples include also diffraction by an edge and Fourier properties of a step function.
\end{abstract}

(C)2003 Optical Society of America

OCIS codes: (000.2060) Education, (350.5500) Propagation

\section{Introduction}

Understanding optics with the use of mechanical analogies has its rich history and includes the works by Fermat, Newton, Hamilton, Jacoby, WKB, V. Arnold, M. V. Berry and by many other remarkable physicists and mathematicians. These analogies are very useful for teaching optics as well, [1-3]. This is especially true with respect to polarizational phenomena.

\section{Basic device and experiments to be performed}

A bi-frequency pendulum on a rotary platform was used by us for more than 10 years, with gradual addition of new demonstrations. It may be easily assembled out of "Lazy Susan" kitchenware with the use of standard half-inch steel plumber's pipes and flanges and a plumb-bob as a weight. We have previously described the use of this mechanical model for teaching optical birefringence. This talk deals with more detailed application of the device for teaching adiabatic following and other optical effects with clear physical interpretation and rather subtle mathematics of their description.

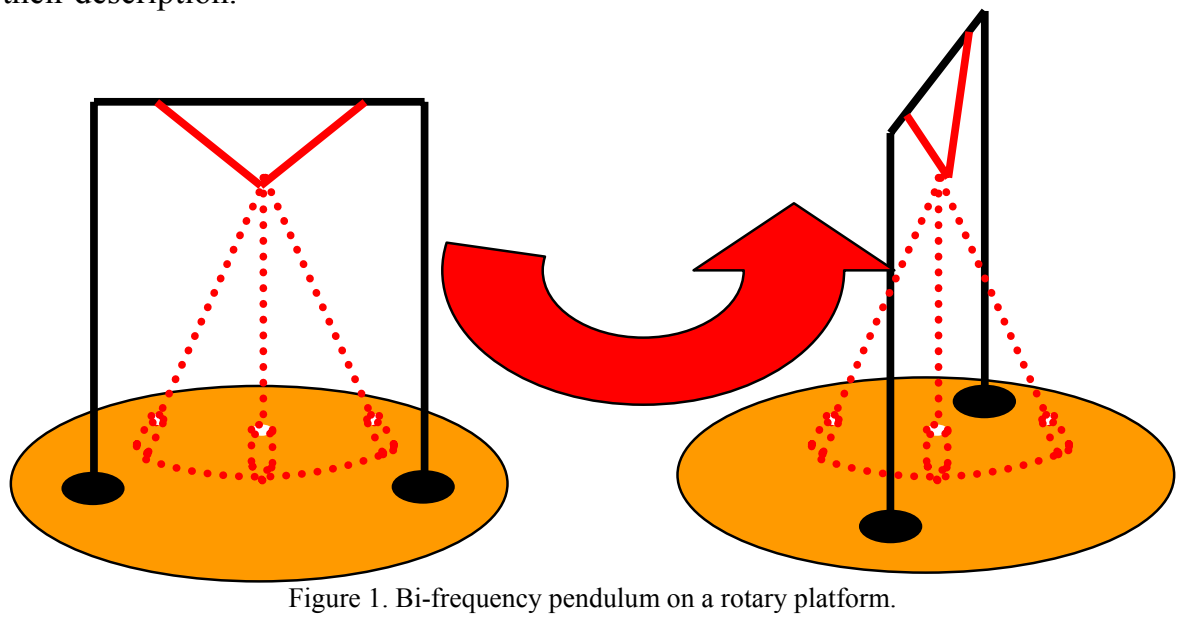

Adiabatic following. Start the rotation of the platform gradually: much less than by $90^{\circ}$ during the time equal to the period of beats. Then one sees a surprising result: the original "linear polarization" stays linear and is considered to follow adiabatically the rotation of the frame. [By the way, one should reject the recollections about the use of the word "adiabatically" in thermodynamics.] The same result is equally valid with the other linearly polarized eigenmode. The important thing is the smoothness and slowness of switching the rotation "on" and "off." During the rotation, in-between the "on" and "off", polarization becomes slightly elliptical, but at the end it will be restored to the original linear type of polarization, to the original eigenmode.

There are extra experiments preparing the audience to the explanation of the adiabatic following regime. One should start a linearly polarized (e.g. low-frequency) eigenmode, and then make fast platform rotation at a small angle, e.g. at $15^{\circ}$. The performer should point audience's attention to excitation of the other mode (x-mode). This excitation, even being small, reveals itself clearly by the presence of beats. Launch the pure low-frequency eigenmode again, at this new position of the platform, and again make fast platform rotation in the same direction, at the same small angle and with the same result. 
Here is the explanation of adiabatic following that is both scientifically correct and may be understood at the lowest level of mathematics comprehension. Under slow rotation, the device continuously "tries" to excite the "wrong" mode, see the above preparatory experiments. However, it tries to excite the high-frequency mode, using for that purpose the low-frequency excitation force from the actual oscillations present. But the excitation out of resonance is known to be in-effective. Therefore the "wrong" mode is not excited to any considerable level. There is one-to-one correspondence between this seemingly primitive explanation and the Slowly Varying Envelope Approximation (SVEA) of the Maxwell equations in twisted anisotropic medium. Therefore, this explanation is worth digesting; it introduces the general notion of adiabatic following.

Slightly elliptical "polarization" that appears in the process of adiabatic following may be interpreted as manifestation of the character of low-frequency eigenmode in the presence of both, bi-pivotal attachment of the pendulum's threads, as in Figure 1, and Coriolis force in the rotating frame. Relation of this weak ellipticity to Fourier transform of a step function and to diffraction of light by an edge will be discussed in the talk.

\section{Conclusion}

To conclude, we demonstrated the performance of inexpensive and effective device, bi-frequency pendulum on a rotary platform, for teaching optical phenomena. Those include adiabatic following of polarization in Liquid Crystals and the mechanism of work of Liquid Crystal Displays.

\section{References}

1. M. Born, E. Wolf, Principles of Optics, 7-th ed., Cambridge U. Press, 1999.

2. E Hecht, Optics, 3-rd ed., Addison-Wesley, Reading, MA, 1998.

3. R. W. Wood, Physical Optics, 3-rd ed., OSA, Washington DC, 1988. 